\title{
A retrospective study of the spectrum of fungal keratitis in southeastern China
}

\author{
Yan Lin ${ }^{1}$, Jingjin Zhang ${ }^{1}$, Xiaoli Han ${ }^{1}$, Jianzhang $\mathrm{Hu}^{2}$ \\ ${ }^{1}$ Department of Ophthalmology, the First Affiliated Hospital of Fujian Medical University, Fuzhou, China; ${ }^{2}$ Department of Ophthalmology, Fujian \\ Medical University Union Hospital, Fuzhou, China \\ Contributions: (I) Conception and design: J Hu; (II) Administrative support: X Han; (III) Provision of study materials or patients: J Hu; (IV) Collection \\ and assembly of data: J Zhang; (V) Data analysis and interpretation: Y Lin; (VI) Manuscript writing: All authors; (VII) Final approval of manuscript: \\ All authors. \\ Correspondence to: Jianzhang Hu. Department of Ophthalmology, Fujian Medical University Union Hospital, 29 Xinquan Road, Fuzhou 350005, \\ China. Email: ophhjz@163.com.
}

Background: This study investigated the epidemiological characteristics, predisposing factors, clinical features, microbiological findings, and treatment outcomes of patients with fungal keratitis in southeastern China.

Methods: A retrospective review was carried out on 718 patients diagnosed with fungal keratitis at the the First Affiliated Hospital of Fujian Medical University between January 2004 and December 2017. The sociodemographic data, predisposing factors, clinical details, microbiological findings, and treatment strategies were analyzed.

Results: Fungal keratitis was diagnosed in 718 patients (442 male and 276 female; mean age, $41.4 \pm 13.1$ years). Most patients came from rural areas (79.7\%) and farm work was the main occupational activity $(51.7 \%)$. Cases were more common during the harvest season between October and December (41.6\%). Corneal trauma $(73.7 \%)$, particularly injury with vegetative matter $(51.5 \%)$, was the predominant predisposing factor. Corneal scrapings obtained from 621 patients were diagnosed as positive on direct microscopy using a $10 \%$ potassium hydroxide $(\mathrm{KOH})$ wet mount preparation. The positive culture rate of corneal scrapings was $89.6 \%$. Fungal isolates were Fusarium species in 444 eyes and Aspergillus species in 98 eyes. Antifungal medications were used to treat 529 patients and 189 patients received surgery.

Conclusions: Fungal keratitis is a leading cause of infective corneal ulcers in southeastern China. Corneal trauma was the major predisposing factor and direct microscopic examination was a rapid and sensitive method for diagnosis. The species Fusarium was the most common fungal isolate. Antifungal medication was an effective method for treating early and mild cases.

Keywords: Spectrum; epidemiology; fungal keratitis; southeastern China

Submitted Jun 21, 2021. Accepted for publication Sep 01, 2021.

doi: 10.21037/apm-21-1949

View this article at: https://dx.doi.org/10.21037/apm-21-1949

\section{Introduction}

Fungal keratitis is a major cause of blindness in developing countries such as China, India, Ghana, and Nepal (1-4). The increased prevalence of fungal keratitis over the past two decades is believed to be related to the growing number of cases of ocular trauma, the widespread abuse of broad-spectrum antibiotics, corticosteroids, and immunosuppressants, and the increasing use of corneal contact lenses. Long-term use of immunosuppressive agents can cause low resistance and cause fungal keratitis infection.

Heightened awareness of the problem among ophthalmologists and growing familiarity with diagnostic methods have also contributed to the increased recognition of this disease. 
The epidemiological features of fungal keratitis vary with respect to geographic locale and climate conditions (3). In recent years, with the continuous change of regional environment and climate, It should be a matter of concern that whether the trend of fungal keratitis and the distribution of pathogenic fungi have changed. China is a vast country, covering from tropical to sub-Arctic, this article wants to explore the fungal keratitis causes in different climate, although there are many retrospective study of fungal keratitis in China, but epidemiological data on keratitis in southeastern is sparse.

In studies undertaken worldwide, species of Aspergillus and Fusarium were found to be the predominant fungi, vegetal trauma was the main risk factor, and fungal culture of the specimen was necessary for effective therapy. A recent study in northern China by Xie et al. analyzed the epidemiological features and laboratory findings in patients with fungal keratitis (1). However, comparable data for southeastern China is poor. Southeastern China is a typical subtropical area with hilly terrain and a primarily agrarian population, which are additional predisposing factors for fungal keratitis. This study aimed to summarize the epidemiological features, laboratory findings, and treatment outcomes of 718 cases of fungal keratitis (718 eyes) in southeastern China and provide a useful guide for locally practicing ophthalmologists. We present the following article in accordance with the STROBE reporting checklist (available at https://dx.doi.org/10.21037/apm-21-1949).

\section{Methods}

\section{Patients}

This hospital-based retrospective study involved 1,463 consecutive patients ( 1,463 eyes) who presented with infective corneal ulcers to the inpatient department of the Eye Center at the First Affiliated Hospital of Fujian Medical University between January 2004 and December 2017. As the principal referral eye center in southeastern China, this institution serves a large proportion of patients with eye diseases in Fujian Province and neighboring provinces. All procedures performed in this study involving human participants were in accordance with the Declaration of Helsinki (as revised in 2013). The study was approved by Ethics Committee of the First Affiliated Hospital of Fujian Medical University (No.: ICE-FOM-013-1.0). Individual consent for this retrospective analysis was waived. Institutional review board approval was obtained.
A detailed history from each patient with a clinical diagnosis of corneal ulcer was recorded, including the age, sex, occupation, onset of symptoms, predisposing risk factors, clinical details, prior treatment modalities, and visual outcomes. An ocular examination of both eyes was performed using slit-lamp biomicroscopy. The visual acuity at presentation, symptoms, and the size and depth of the stromal infiltrate were documented. In addition, the depth of the lesion, the presence or absence of hypopyon, and the status of the anterior chamber were evaluated.

\section{Diagnostic criteria}

A definitive diagnosis of fungal keratitis was made if: (I) corneal scrapings incubated with potassium hydroxide $(\mathrm{KOH})$ and examined on wet mounts revealed fungal elements in smears; (II) fungus grew in more than one medium when corneal scrapings were subjected to fungal culture and strain identification; (III) histopathologic examination revealed fungal presence $(1,5)$.

\section{Collection of specimens and laboratory procedures}

Corneal scrapings were aseptically collected from the leading edge or base of the ulcer using a disposable microblade, except in cases of corneal perforation. A portion of each scraping was examined microscopically for the presence of fungi, bacteria, or Acanthamoeba by staining with $10 \% \mathrm{KOH}$ or Gram stain. Another portion was subjected to fungi, bacteria, or Acanthamoeba culture. A fungal culture was considered positive using direct microscopy, and isolates were identified based on their macroscopic and microscopic colonial morphology.

\section{Treatment protocol}

Antifungal medications comprising $0.5 \%$ fluconazole combined with $0.25 \%$ amphotericin B or $0.5 \%$ fluconazole combined with $5 \%$ natamycin were administered. One drop was administered every 30 minutes while the patients were awake. Patients were also treated with $200 \mathrm{mg}$ of oral itraconazole daily for 21 days. If the corneal infection was not controlled or it continued to deteriorate after more than 7 days of intensive antifungal therapy, a surgical intervention was performed. Surgical interventions included therapeutic penetrating keratoplasty (PK), therapeutic lamellar keratoplasty (LK), and keratectomy in combination with a conjunctival flap. 
Table 1 Predisposing factors for fungal keratitis

\begin{tabular}{lcc}
\hline Predisposing factors & No. of cases & Percentage \\
\hline Trauma & 456 & 73.7 \\
Contact lens wear & 13 & 2.1 \\
Ocular problems & 59 & 9.5 \\
Chronic dacryocystitis & 14 & 2.3 \\
Dry eyes & 11 & 1.8 \\
Herpes simplex keratitis & 11 & 1.8 \\
Exposure keratitis & 7 & 1.1 \\
Post-surgical & 12 & 1.9 \\
Others & 4 & 0.6 \\
Systemic conditions & 75 & 12.1 \\
Diabetes mellitus & 56 & 9.0 \\
Pneumonia & 7 & 1.1 \\
Rheumatoid arthritis & 8 & 1.3 \\
Other & 4 & 0.6 \\
Topical steroids & 16 & 2.6 \\
Total & 619 & 100 \\
\hline
\end{tabular}

After surgery, antifungal chemotherapeutic treatment was continued for 2 weeks, tapering thereafter. All patients were evaluated daily in the first postoperative week, once every other day in the second week, and weekly thereafter for at least 8 months. The follow-up ranged from 8 to 48 months. Each evaluation included graft clarity, late fungal recurrence, visual acuity, and intraocular pressure assessment.

\section{Statistical analysis}

Statistical analysis was carried out using the paired $\chi^{2}$ test and the chi square test with the software package Graphpad Prism 6.0 (Graphpad Software Inc., San Diego, CA, USA).

\section{Results}

\section{Epidemiological characteristics}

Overall, 1,463 patients with a clinical diagnosis of corneal ulcer were enrolled in this study. Of these patients, 718 $(49.1 \%)$ were diagnosed with fungal keratitis and 379 (25.9\%) patients had bacterial keratitis. No pathogens could be isolated in the other 279 patients (19.1\%).
Of the 718 patients with fungal keratitis, $442(61.6 \%)$ patients were male and $276(38.4 \%)$ were female. With a male-to-female ratio of 1.6:1, males were significantly more affected than females $(\mathrm{P}<0.01)$. Patient age ranged from 9 to 80 years. The majority of patients were middle-aged, with the average age at presentation $41.4 \pm 13.1$ years. Most patients $(572 ; 79.7 \%)$ were from rural areas $(\mathrm{P}<0.0001)$. A majority of the patients $(371 ; 51.7 \%)$ were farm workers $(\mathrm{P}<0.0001)$, and $117(16.3 \%)$ patients were physical laborers. Fungal keratitis cases were more common from October to December (299; 41.6\%), followed by July to September $(182 ; 25.3 \%)$. The duration from the onset of symptoms to presentation to our institution ranged from 4 to 130 days (mean, 25.6 \pm 9.7 days), with most patients (529, $73.7 \%$ ) presenting within 2 weeks. Before the initial visit to our institution, primary care was sought by 637 (88.7\%) patients.

\section{Predisposing factors}

Corneal trauma $(456 ; 73.7 \%)$ was identified as the predominant predisposing factor. Contact lens use was a risk factor in $13(2.1 \%)$ cases. Ocular problems that predisposed patients to a corneal ulcer were present in $59(9.5 \%)$ patients. Systemic conditions with possible immunosuppressive consequences included diabetes mellitus, pneumonia, and rheumatoid arthritis. Topical corticosteroids were prescribed to $2.6 \%$ of patients (16/619) before keratitis onset (Table 1).

\section{Clinical features}

The typical clinical features of fungal keratitis include a thick, dry, raised surface and stromal infiltrates with feathery margins. Satellite lesions, immune rings, hypopyon, and endothelial plaques were observed in $16 \%, 11 \%, 37 \%$, and $9 \%$ of patients, respectively. The diameter of corneal ulcers in our cases ranged from 2 to $11 \mathrm{~mm}$ (mean, $5.2 \pm 1.9 \mathrm{~mm}$ ). Corneal perforation was present in 79 (11\%) patients, 198 $(27.6 \%)$ patients had elevated intraocular pressure, and 47 (6.5\%) patients had endophthalmitis.

\section{Microbiological findings}

Corneal scrapings obtained from 652 (90.8\%) of the 718 eyes with fungal keratitis were positive for fungal elements on direct microscopy with $10 \% \mathrm{KOH}$ wet mount or Gramstained smear (Table 2). 
Table 2 Correlation between direct microscopic diagnosis and culture-based diagnosis as well as clinical diagnosis

\begin{tabular}{|c|c|c|c|c|c|c|}
\hline Name of the investigation & Results & No. & \multicolumn{2}{|c|}{ Presence of fungal growth in culture } & Sensitivity (\%) & Specificity (\%) \\
\hline \multirow{3}{*}{$\begin{array}{l}\text { Detection of fungal filaments in } \\
10 \% \mathrm{KOH} \text { smear }\end{array}$} & Positive & 621 & 614 & 7 & 95.5 & 90.7 \\
\hline & Negative & 97 & 29 & 68 & & \\
\hline & Total & 718 & 643 & 75 & & \\
\hline \multirow{2}{*}{$\begin{array}{l}\text { Detection of fungal filaments in } \\
\text { Gram-stained smear }\end{array}$} & Negative & 159 & 89 & 70 & & \\
\hline & Total & 718 & 643 & 75 & & \\
\hline \multirow{2}{*}{$\begin{array}{l}\text { Clinical suspicion of fungal } \\
\text { keratitis on slit-lamp examination }\end{array}$} & Positive & 649 & 608 & 41 & 94.6 & 45.3 \\
\hline & Negative & 69 & 35 & 34 & & \\
\hline
\end{tabular}

Table 3 Spectrum of fungal species in southeastern China

\begin{tabular}{|c|c|c|}
\hline Species & No. of isolates & Percentage \\
\hline Fusarium species & 444 & 69.1 \\
\hline F. solani & 197 & 30.6 \\
\hline F. moniliforme & 52 & 8.1 \\
\hline F. oxysporum & 53 & 8.2 \\
\hline F. nivale & 121 & 18.8 \\
\hline Other & 21 & 3.3 \\
\hline Aspergillus species & 98 & 15.2 \\
\hline A. flavus & 37 & 5.8 \\
\hline A. fumigatus & 31 & 4.8 \\
\hline A. terreus & 12 & 1.9 \\
\hline A. niger & 7 & 1.1 \\
\hline A. oryzae & 5 & 0.8 \\
\hline Other & 6 & 0.9 \\
\hline Alternaria species & 34 & 5.3 \\
\hline Penicillium species & 16 & 2.5 \\
\hline Candida albicans & 14 & 2.2 \\
\hline Microsporum & 11 & 1.7 \\
\hline Sporothrix schenckii & 4 & 0.6 \\
\hline Other & 22 & 3.4 \\
\hline Total & 643 & 100.0 \\
\hline
\end{tabular}

Cultures were positive in 827 corneal ulcers. Of the 643 fungal culture-positive cases, species of Fusarium was the most frequent isolate [444 (69.1\%)], followed by Aspergillus [98 (15.2\%)], Alternaria, Penicillium, Candida albicans, Microsporum, and Sporothrix schenckii. There were also 22 unidentified cultured specimens (Table 3).

\section{Treatment outcome}

Antifungal medications were used to treat 529 patients while surgery was used in 189 patients, including therapeutic PK in 61 (32.2\%) patients, therapeutic LK in 35 (18.5\%) patients, keratectomy in combination with a conjunctival flap in 67 (35.4\%) patients, and evisceration in $26(13.8 \%)$ patients. Follow-up ranged from 8 to 48 months. Halfway through treatment, 21 patients were lost to follow-up.

Combined antifungal medications were effective for early and mild cases of fungal keratitis. Of the cases cured with medication, the course duration ranged from 19 to 96 days (mean, $27 \pm 8.9$ days), showing therapeutic effect within the first 5 to 14 days. Secondary glaucoma occurred in 38 cases, requiring trabeculectomy. A corneal leucoma formed in 216 cases (40.8\%), a corneal macula formed in 192 cases (36.3\%), and an adherent leucoma formed in 43 cases (8.1\%).

Fungal infection recurred in $4(11.4 \%)$ of the patients who received LK. The recurrent keratitis was controlled with PK, and the grafts remained clear at follow-up. Of the cases receiving PK, keratitis recurred in $9(14.8 \%)$ 
patients. Repeat grafting was performed for 2 of these cases, 3 required conjunctival flaps, 3 cases eventually required evisceration for control of the disease, and one was lost to follow-up.

\section{Discussion}

The incidence of fungal keratitis in our study was similar to that reported in northern China (1). Research results from different parts of the world indicate that the incidence of fungal keratitis ranges from $6 \%$ to $56 \%(6,7)$. A Taiwanese study found that fungal pathogens were isolated from only $13.5 \%$ of 476 eyes with microbial keratitis (8). This disparity could be due to the use of more mechanized production modes in Taiwan compared to those used in southeastern China.

Farmers were the most common patients with fungal keratitis (1-3). In our study, $51.7 \%$ of patients were farm workers and most patients were from rural areas. This corresponds to the sex and age of patients in this study. In southeastern China, particularly in Fujian province, agriculture is the primary industry and the labor force mainly comprises young to middle-aged men. Correspondingly, fungal keratitis cases were more common between October and December due to the high humidity and temperature difference between day and night during these months. This type of environment results in a propensity for fungal growth. Farm workers are often very busy during the harvest season and are prone to vegetative injuries.

Early diagnosis and early treatment are critical for the treatment of fungal keratitis (9). In our study, most patients visited the hospital within 15 days after the onset of symptoms, indicating that in southeastern China, patient health awareness had significantly improved with rapid economic development. We found that the longer the duration of symptoms and the longer the delay to obtaining medical care, the worse the response to antifungal agents, the more likely it was that patients underwent corneal surgery, and the greater likelihood of sequelae. The differential diagnoses include bacterial keratitis. Bacterial keratitis is usually caused by bacterial infection after trauma. There are many kinds of Species of pathogenic fungi in fungal keratitis, among which fusarium and aspergillus are the most common. Bacterial keratitis is a urgent disease, which occurs usually between 24 and 48 hours after the occurrence of trauma, the common symptoms are redness, photophobia, tears, decreased vision, increased secretion, etc. Fungal keratitis is slower, but with higher blinding rate. The patient's corneal infiltration can easily cause endophthalmitis.

Corneal trauma was clearly identified as the predominant predisposing factor in our study. This is similar to the findings from northern China and from many agricultural producing countries, such as India and Nepal $(1,2,4)$. In previous studies, the percentage of corneal trauma has been reported to range from $8 \%$ to $66 \%(6,10)$, with agricultural vegetation identified as the most frequent cause of injury ( $44 \%$ to $69 \%$ of cases) $(6,9,11)$. Contact lens wear was reported to be a major risk factor in many studies $(2,12)$ but was much lower in our study as farmers in China seldom wear contact lenses. Topical corticosteroid use was also reported to be correlated with the incidence and course of fungal keratitis $(2,13,14)$. In our study, those patients who used topical steroids had more extensive infiltrates and the response to antifungal therapy was slower. Therefore, we agreed that corticosteroids should be contraindicated in patients with fungal keratitis $(5,13,15)$.

In the present study, the positive rate of fungal filaments on direct microscopic examination was $90.8 \%$, which is consistent with previous studies from northern China (88.7\%) (1) and Northwest India (90\%) (11). The sensitivity of $10 \% \mathrm{KOH}$ wet mount preparation was higher than the sensitivity of a Gram-stained smear in this study, which is similar to previous reports $(11,16)$. We concluded that direct microscopic examination with a $10 \% \mathrm{KOH}$ wet mount should be one of the main methods for early diagnosis of fungal keratitis; it is convenient, rapid, inexpensive, highly sensitive, and highly specific. We also believe it is important for doctors to master specific skills for corneal scraping, which include removing the surface secretions and necrotic tissue first, then repeatedly scraping the corneal ulcers at the bottom and edge, and finally smearing a thin layer on a wet mount. This helps to show the fungal hyphae, spores, and Acanthamoeba more clearly.

The positive culture rate in corneal scrapings was $89.6 \%$ in this study, which is similar to previous studies $(1,3)$. A fungal culture is considered the 'gold standard' for the diagnosis of fungal infections. However, this process often requires more than 5 days and thus is not useful for early diagnosis. Patients with a suspected case of fungal keratitis should simultaneously have a direct microscopic examination and a fungal culture to avoid a delayed diagnosis.

The most common fungus reported in China is Fusarium, which accounts for $58.7 \%$ of cases, similar to the 
findings of our study $(1,2)$. The climate of Southeastern China is different from that of northern China, and there is no obvious difference between the seasons. The temperature and humidity are relatively higher, which can contribute to the growth of fungi. We want to explore the causes of fungal keratitis in different climates, but the data show that the main pathogenic bacteria is Fusarium. In this study, Fusarium was the most frequent isolate, followed by Aspergillus. Fusarium has also been found to be the predominant species in Southern India, Florida, Paraguay, Nigeria, Tanzania, Hong Kong, and Singapore $(6,7,11,13,17-22)$. However, in Northern India, Nepal, and Bangladesh, Aspergillus is the most frequent cause of fungal keratitis $(2,4,23)$. This phenomenon may be explained by the differences in demographic characteristics, climate, and the natural environment. Filamentous fungi are more frequently encountered than yeasts in tropical and subtropical regions. Fusarium grows in cornea horizontally, diagonally and vertically, and the hyphae are easy to spread and multiply around the lesion while Aspergillus grow in cornea diagonally and vertically. Filamentous fungi can penetrate corneal stroma and into anterior room, invade iris and the tissue inside the eye, cause fungal iridocyclitis, intraocular inflammation, and complicated cataract. The latter has been isolated with greater frequency in the west where traumatic keratomycosis is rare $(7,13)$. Data from developed nations such as the United States and Britain have shown that Candida is the most common isolate in cases of fungal keratitis (24). It is thought that yeastinfectious keratitis usually occurs in host corneas with compromised immune function. In our study, the 8 cases of Candida keratitis were observed in patients with diabetes mellitus.

In the present study, 529 cases were treated with antifungal medications. The cure rate was $73.7 \%$, which was much higher than the cure rate reported in northern China (7.6\%). In southeastern China, there are few options beyond medical treatment for fungal keratitis due to the lack of donor corneas. As a result, we have accumulated some experience with antifungal medications, including: (I) combining drugs, such as itraconazole can combine with fluconazole; (II) coordinating debridement at intervals of 5 to 7 days to remove necrotic tissue; (III) adjusting the frequency of medications according to the patient's condition and reducing the frequency of medicine administration as the corneal ulcer gradually heals; (IV) adherence to and the cautious withdrawal of medications are also critical. It was proposed that the use of antifungal medications is sustained until the corneal ulcer has healed completely and to adhere to medications for a duration of 1 month without exacerbations.

If an infection failed to respond to antifungal medical therapy, an operation was performed. Keratoplasty was a popular method, although it occasionally led to fungal recurrence and postoperative immune rejection, which was similar to the findings of some previous studies (25). Conjunctival flap-covering surgery often causes corneal leucoma, new vessels, and poor vision (26). However, ophthalmologists usually prefer this method because of the lack of donor corneas.

The main clinical feattire of fungal keratitis is hyphal coating. Fusaria are the most commonpathogenic bacteria, and the peak onset periods were during autumn and summer harvests. Fully understand the epidemiological characteristics of fungal keratitis, early diagnosis and early treatment are of great significance for prognosis.

\section{Conclusions}

In summary, this study focused on the epidemiological and etiological data obtained from a large cohort of patients with fungal keratitis in southeastern China and provides significant insights into the understanding of this potentially devastating corneal disease. In southeastern China, the Fusarium species was the most commonly isolated pathogen, agricultural activity was the principal causative factor, and a large proportion of patients received early treatment and were cured by antifungal medications. We hope that our study may help our colleagues diagnose and properly treat this disease and achieve a better outcome for patients.

\section{Acknowledgments}

Funding: None.

\section{Footnote}

Reporting Checklist: The authors have completed the STROBE reporting checklist. Available at https://dx.doi. org/10.21037/apm-21-1949

Data Sharing Statement: Available at https://dx.doi. org/10.21037/apm-21-1949

Conflicts of Interest: All authors have completed the ICMJE 
uniform disclosure form (available at https://dx.doi. org/10.21037/apm-21-1949). The authors have no conflicts of interest to declare.

Ethical Statement: The authors are accountable for all aspects of the work in ensuring that questions related to the accuracy or integrity of any part of the work are appropriately investigated and resolved. All procedures performed in this study involving human participants were in accordance with the Declaration of Helsinki (as revised in 2013). The study was approved by Ethics Committee of the First Affiliated Hospital of Fujian Medical University (No.: ICE-FOM-013-1.0). Individual consent for this retrospective analysis was waived.

Open Access Statement: This is an Open Access article distributed in accordance with the Creative Commons Attribution-NonCommercial-NoDerivs 4.0 International License (CC BY-NC-ND 4.0), which permits the noncommercial replication and distribution of the article with the strict proviso that no changes or edits are made and the original work is properly cited (including links to both the formal publication through the relevant DOI and the license). See: https://creativecommons.org/licenses/by-nc-nd/4.0/.

\section{References}

1. Xie L, Zhong $W$, Shi $W$, et al. Spectrum of fungal keratitis in north China. Ophthalmology 2006;113:1943-8.

2. Chowdhary A, Singh K. Spectrum of fungal keratitis in North India. Cornea 2005;24:8-15.

3. Lv J, Zhang K, Chen Q, et al. Deep learning-based automated diagnosis of fungal keratitis with in vivo confocal microscopy images. Ann Transl Med 2020;8:706.

4. Upadhyay MP, Karmacharya PC, Koirala S, et al. Epidemiologic characteristics, predisposing factors, and etiologic diagnosis of corneal ulceration in Nepal. Am J Ophthalmol 1991;111:92-9.

5. Xie L, Hu J, Shi W. Treatment failure after lamellar keratoplasty for fungal keratitis. Ophthalmology 2008;115:33-6.

6. Srinivasan M, Gonzales CA, George C, et al. Epidemiology and aetiological diagnosis of corneal ulceration in Madurai, south India. Br J Ophthalmol 1997;81:965-71.

7. Thomas PA. Mycotic keratitis--an underestimated mycosis. J Med Vet Mycol 1994;32:235-56.

8. Fong CF, Tseng CH, Hu FR, et al. Clinical characteristics of microbial keratitis in a university hospital in Taiwan. Am J Ophthalmol 2004;137:329-36.

9. Polack FM, Kaufman HE, Newmark E. Keratomycosis. Medical and surgical treatment. Arch Ophthalmol 1971;85:410-6.

10. Tanure MA, Cohen EJ, Sudesh S, et al. Spectrum of fungal keratitis at Wills Eye Hospital, Philadelphia, Pennsylvania. Cornea 2000;19:307-12.

11. Panda A, Sharma N, Das G, et al. Mycotic keratitis in children: epidemiologic and microbiologic evaluation. Cornea 1997;16:295-9.

12. Rosa RH Jr, Miller D, Alfonso EC. The changing spectrum of fungal keratitis in south Florida. Ophthalmology 1994;101:1005-13.

13. Liesegang TJ, Forster RK. Spectrum of microbial keratitis in South Florida. Am J Ophthalmol 1980;90:38-47.

14. Berson EL, Kobayashi GS, Becker B, et al. Topical corticosteroids and fungal keratitis. Invest Ophthalmol 1967;6:512-7.

15. Jones BR. Principles in the management of oculomycosis. XXXI Edward Jackson memorial lecture. Am J Ophthalmol 1975;79:719-51.

16. Vajpayee RB, Angra SK, Sandramouli S, et al. Laboratory diagnosis of keratomycosis: comparative evaluation of direct microscopy and culture results. Ann Ophthalmol 1993;25:68-71.

17. Erie JC, Nevitt MP, Hodge DO, et al. Incidence of ulcerative keratitis in a defined population from 1950 through 1988. Arch Ophthalmol 1993;111:1665-71.

18. Miño de Kaspar H, Zoulek G, Paredes ME, et al. Mycotic keratitis in Paraguay. Mycoses 1991;34:251-4.

19. Gugnani HC, Talwar RS, Njoku-Obi AN, et al. Mycotic keratitis in Nigeria. A study of 21 cases. Br J Ophthalmol 1976;60:607-13.

20. Mselle J. Fungal keratitis as an indicator of HIV infection in Africa. Trop Doct 1999;29:133-5.

21. Houang E, Lam D, Fan D, et al. Microbial keratitis in Hong Kong: relationship to climate, environment and contact-lens disinfection. Trans R Soc Trop Med Hyg 2001;95:361-7.

22. Wong TY, Fong KS, Tan DT. Clinical and microbial spectrum of fungal keratitis in Singapore: a 5-year retrospective study. Int Ophthalmol 1997;21:127-30.

23. Williams G, Billson F, Husain R, et al. Microbiological diagnosis of suppurative keratitis in Bangladesh. Br J Ophthalmol 1987;71:315-21.

24. Yamamoto GK, Pavan-Langston D, Stowe GC 3rd, et 
al. Fungal invasion of a therapeutic soft contact lens and cornea. Ann Ophthalmol 1979;11:1731-5.

25. Xie L, Dong X, Shi W. Treatment of fungal keratitis by penetrating keratoplasty. Br J Ophthalmol 2001;85:1070-4.

26. Mizunoya S, Watanabe Y. Paecilomyces keratitis with

Cite this article as: Lin $\mathrm{Y}$, Zhang J, Han X, Hu J. A retrospective study of the spectrum of fungal keratitis in southeastern China. Ann Palliat Med 2021;10(9):9480-9487. doi: 10.21037/apm-21-1949 corneal perforation salvaged by a conjunctival flap and delayed keratoplasty. Br J Ophthalmol 1994;78:157-8.

(English Language Editor: A. Muijlwijk) 